\title{
The new technology of precise anatomical sublobar resection by using 3D medical image analyzer and a fluorescence guided surgery with transbronchial injection of indocyanine green
}

\author{
Yasuo Sekine, Eitetsu Koh, Hodaka Oheda, Hidehisa Hoshino \\ Departments of Thoracic Surgery, Tokyo Women's Medical University Yachiyo Medical Center, Chiba, Japan \\ Contributions: (I) Conception and design: Y Sekine; (II) Administrative support: Y Sekine; (III) Provision of study materials or patients: E Koh, \\ H Oheda, H Hoshino; (IV) Collection and assembly of data: E Koh, H Oheda, H Hoshino; (V) Data analysis and interpretation: Y Sekine; (VI) \\ Manuscript writing: All authors; (VII) Final approval of manuscript: All authors. \\ Correspondence to: Yasuo Sekine, MD, PhD. Department of Thoracic Surgery, Tokyo Women's Medical University Yachiyo Medical Center, 477-96 \\ Owada-Shinden, Yachiyo, Chiba 276-8524, Japan. Email: sekine.yasuo@twmu.ac.jp.
}

\begin{abstract}
Background: The confirmation of an appropriate resection margin from the tumor is crucial for reducing the risk of local recurrence after sublobar resection for pulmonary malignancies.

Methods: From August 2014 to December 2017, 66 locations in 64 patients (primary lung cancer 48, metastatic lung tumor 15, benign disease 3) were enrolled. In lung cancer, active limited resection was done in 31 and passive limited resection was done in 17. Preoperatively, each patient created several virtual sublobar resections by using 3D Volume Analyzer. We measured the surgical margin in each simulated sublobar resection and selected the most appropriate procedure. Surgical resection matched with virtual sublobar resection was done by using an infrared thoracoscopy with transbronchial indocyanine green (ICG) instillation. In lung cancer, we compared surgical outcomes between ICG cases and 47 historical segmentectomy cases.
\end{abstract}

Results: The types of segmentectomy were subsegmental resection in 5, simple segmentectomy in 15 , complex segmentectomy in 16 and extended segmentectomy in 22 and anatomical super deep wedge resection in 8 . The shortest distance of surgical margin by simulation and an actual measurement were $20.8 \pm 11.1$ and $22.6 \pm 8.3 \mathrm{~mm}$, respectively $(\mathrm{P}=0.186)$. Postoperative recurrence was found in 9 cases (distant in 8 and mediastinal lymph node in 1). No locoregional recurrence was found in all cases. Postoperative recurrence was similar between the two groups in active and passive limited resection, respectively.

Conclusions: ICG-guided sublobar resection by transbronchial ICG instillation is applicable to any type of sublobar resection and can control local recurrence of lung neoplasms.

Keywords: Synapse 3D; sublobar resection; indocyanine green (ICG); fluorescence imaging; lung neoplasms

Received: 12 November 2019; Accepted: 11 December 2019; Published: 05 October 2020.

doi: 10.21037 /jovs.2019.12.12

View this article at: http://dx.doi.org/10.21037/jovs.2019.12.12

\section{Introduction}

Pulmonary sublobar resection has been recognized as an operative option for complete resection of early stage lung cancer (1) and metastatic lung tumor. The standard approach for anatomical lung segmentectomy is vascular and bronchial transections followed by division of intersegmental planes. However, this method has several problems. The identification of segmental bronchi and associated vessels is sometimes difficult and intraoperative inflation of the lung is an obstacle for thoracoscopic visualization. Although the confirmation of an appropriate resection margin from the tumor is crucial for reducing the risk of local recurrence (2), there has been no method of measurement. 


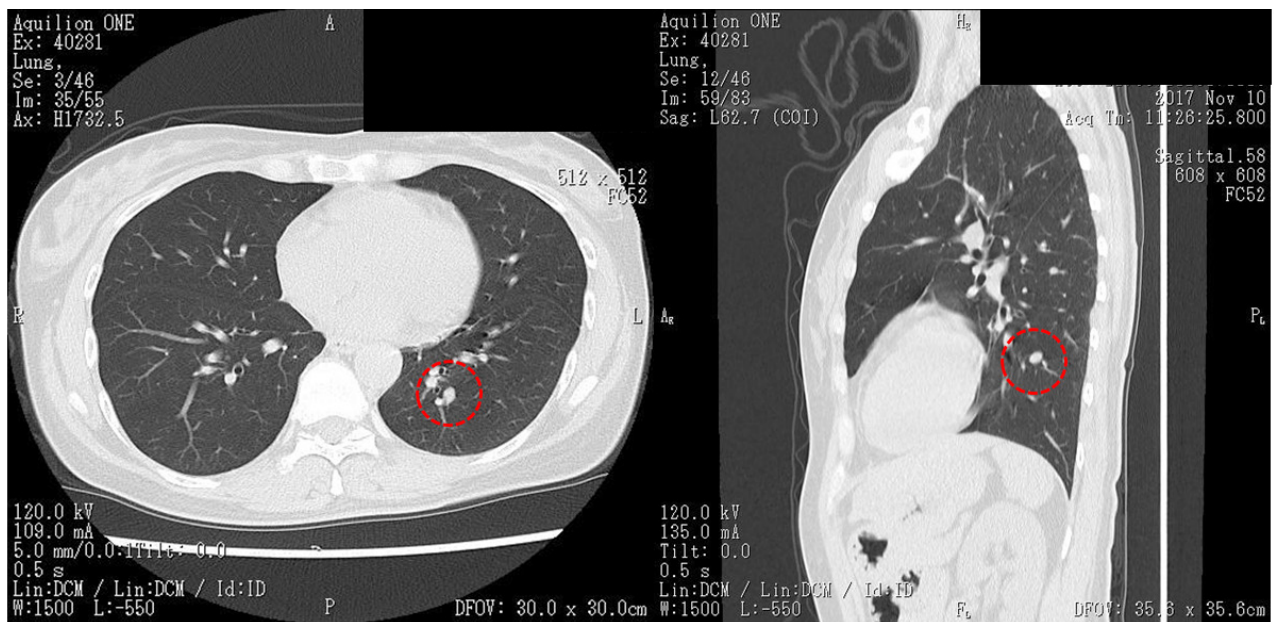

Figure 1 Forty-two-year-old female with left metastatic lung tumor. There is a nodule in the left lower lobe at $S^{10}$ c.

Furthermore, recent advances of computer tomography make the increase of early stage lung cancer detection and various methods of tumor localization marking have been reported in minimally invasive surgery. However, precise tumor localization with enough surgical margin has not been well established.

We previously developed and reported a novel approach for performing segmentectomy by using an infrared thoracoscopy with transbronchial instillation of indocyanine green (ICG) (3). We improved this method by combining with the most advanced computer technology of making a virtual sublobar resection and established a precise anatomical sublobar resection with enough surgical margin. This method can also serve both as tumor localization marking and anatomical deep wedge resection with enough margin for early stage lung cancers and metastatic lung tumors.

\section{Surgical technique}

Before performing operation, high resolution chest computed tomography (CT), three-dimensional (3D) pulmonary angiography and virtual bronchoscopy to confirm associated vessels and bronchi and a couple of simulations of sublobar resection in order to obtain an appropriate resection margin from the tumor were performed. In detail, subjects underwent multislice enhanced CT, using 320-slice scanners (Aquilion ONE ${ }^{\mathrm{TM}}$ / ViSION Edition; Toshiba Medical Systems, Tokyo, Japan) (Figure 1) to create pulmonary angiography and virtual bronchoscopy, to simulate anatomical sublobar resection and to measure lung volume by Volume Analyzer Synapse 3D VINCENT (Fujifilm co., Tokyo, Japan) (Figure 2). We measured the shortest distance from the tumor to the resection margin by VINCENT and selected the most appropriate area of sublobar resection based on the following decision making; resection margin approximately $2 \mathrm{~cm}$ from the tumor or longer than tumor diameter. Nomenclature of lung segments was based on the report by Jafek and Carter (4) and Nomina Anatomica (5).

After induction of general anesthesia, a single-lumen endotracheal tube or a laryngeal mask was introduced for transbronchial ICG instillation. Twenty-five $\mathrm{mg} / 10 \mathrm{~mL}$ of ICG was diluted by $70 \mathrm{~mL}$ of saline and $20 \mathrm{~mL}$ of autologous blood (AB) for making a 10-fold diluted ICG solution. It is well known that adsorption of ICG to human serum albumin can increase the fluorescence intensity (6).

In a supine position, a thin-bronchoscope (BF-P260F, Olympus Medical Co., Tokyo, Japan) was inserted into a targeted bronchus, bronchial catheter with balloon (Olympus disposable balloon catheter B5-2C/2LA, Olympus Medical Co., Tokyo, Japan) was inserted and the balloon was inflated at the orifice of the bronchus. Ten $\mathrm{mL}$ of 10 -fold salineAB-diluted ICG was instilled into each target subsegmental or subsubsegmental bronchus and a $200-400 \mathrm{~mL}$ of air was then supplied into the bronchus to distribute ICG into peripheral regions (Video 1). During this maneuver, the scope was kept fitting into the balloon perfectly for prohibiting ICG leakage and for visualizing the tip position of catheter over the balloon. After ICG instillation, a double-lumen Broncho-cath tube was introduced and $5 \mathrm{cmH}_{2} \mathrm{O}$ of positive end-expiratory pressure ventilation was maintained until the 

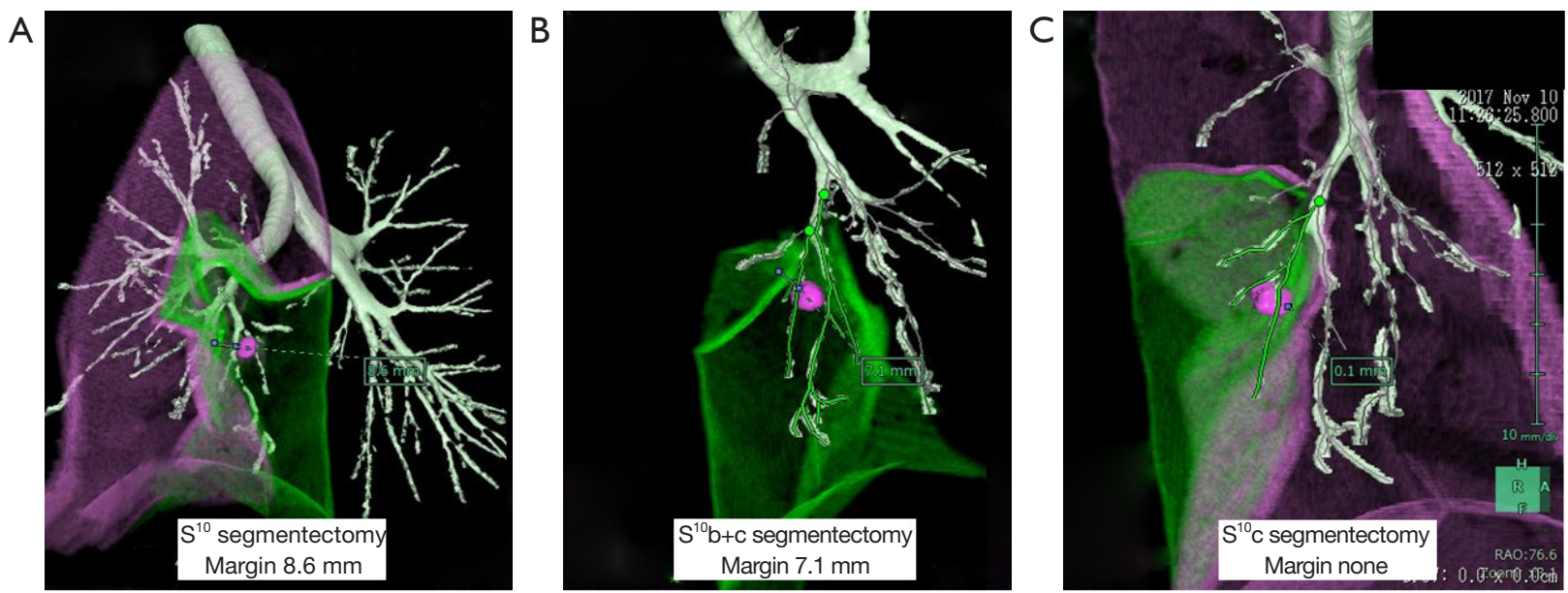

Figure 2 Three virtual segmentectomies were created and the most appropriate resection area was selected based on the surgical margin distance. Left $\mathrm{S}^{10} \mathrm{~b}+\mathrm{c}$ subsegmentectomy was selected. (A) $\mathrm{S}^{10}$; (B) $\mathrm{S}^{10} \mathrm{~b}+\mathrm{c}$; (C) $\mathrm{S}^{10}$ c segmentectomies.

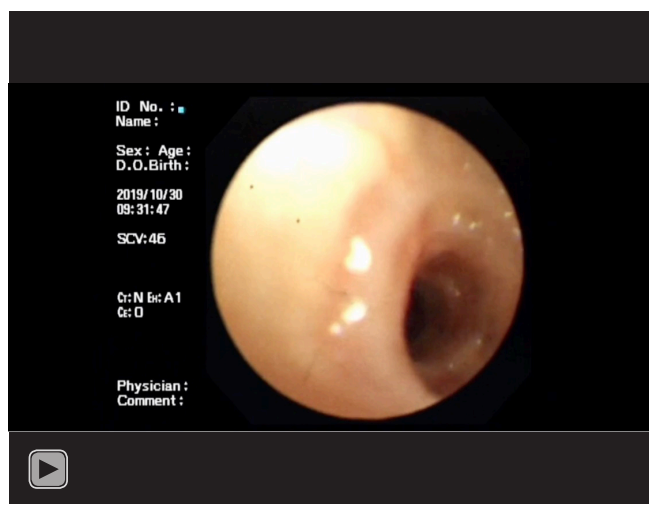

Video 1 Transbronchial ICG injection. Ten $\mathrm{mL}$ of 10 -fold diluted ICG was injected into right B9bii, B9bi and B10bii and $400 \mathrm{~mL}$ of air was injected with balloon inflation at the bronchial orifice, respectively.

start of the operation.

At the beginning of the surgery, the near-infrared (NIR) thoracoscope (PINPOINT, Stryker, MI, USA) was used to visualize the intersegmental lines and planes. The visceral pleura was marked using electric cautery along to the borderline of ICG fluorescence (Figure 3). Simultaneous process of vascular and bronchial division and segmental division can be done because initial identification of segmental planes is possible. Finally, the intersegmental planes were divided by electric cautery and/or endostaplers to complete sublobar resection. After complete resection, infrared thoracoscope could identify no residual tissues which should be resected. Sufficient distance from the

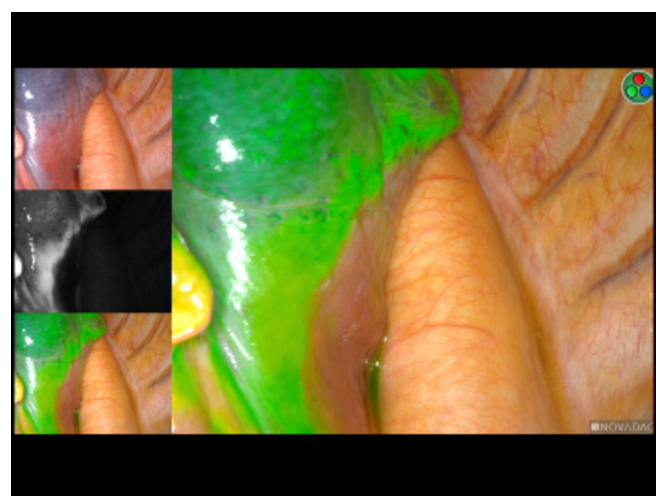

Figure 3 ICG fluorescence is clearly visualized at the initial time of operation. The real image was completely matched with the virtual image. ICG, indocyanine green.

tumor to the resection margin can be measured from the resected specimen (Video 2).

In terms of wedge resection, preoperative virtual subsegmental resection was created in the same manner to fulfill the same condition with segmentectomy. According to this simulation, ICG was instilled into the associated bronchus and wedge resection along to the fluorescence border was accomplished (Video 3).

\section{Methods}

From August 2014 to December 2017, 66 operative cases in 64 patients (primary lung cancer 42, metastatic lung tumor 21 , benign disease 3 ) were enrolled. The study protocol was 


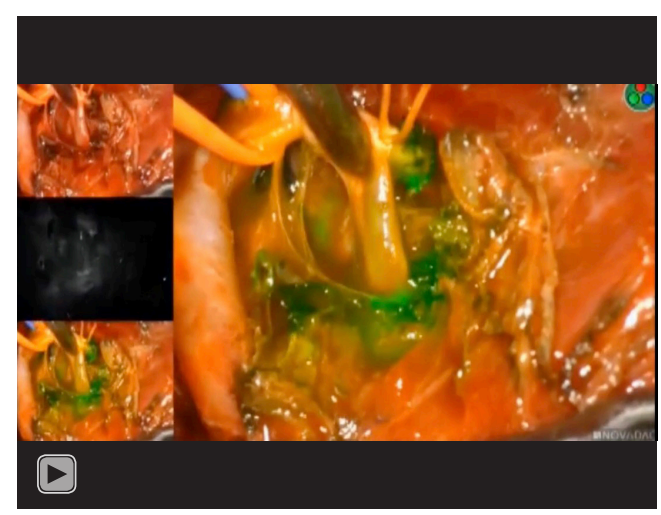

Video 2 lt. S10b+c subsegmentectomy with transbronchial ICG injection into left B10b and B10c for a patient with metastatic lung tumor which is related to Figures 1-4.

approved by the Research Ethics Board of Tokyo Women's Medical University, Japan. Written informed consent was obtained from all patients. The study was conducted in accordance with the Declaration of Helsinki (as revised in 2013).

In lung cancer, active limited resection was done in 29 and passive limited resection was done in 13 . The indication of active limited resection for lung cancer was the maximum diameter of tumor consolidation was $2 \mathrm{~cm}$ or shorter in peripheral region. Passive limited resection was done in patients with low cardiopulmonary function or poor general condition. To evaluate the efficacy of this method in lung cancer (ICG group), we compared with 47 patients (Active limited resection in 24, Passive limited resection in 23) who underwent a traditional thoracoscopic segmentectomy between September 2008 and July 2014 (Control group). VINCENT was not used in all control patients. In the traditional segmentectomy, the target segment was confirmed by using temporary aeration into the associated bronchus. We compared patient clinical data and surgical outcomes between the two groups retrospectively.

Data were analyzed using SPSS Statistics 20 (IBM Japan; Tokyo, Japan). To compare the differences between the ICG and control groups in lung cancer, the Mann-Whitney $\mathrm{U}$ test was utilized to analyze for continuous variables and the Fisher's exact test was used to analyze for categorical variables.

\section{Results}

The types of segmentectomy were subsegmental resection

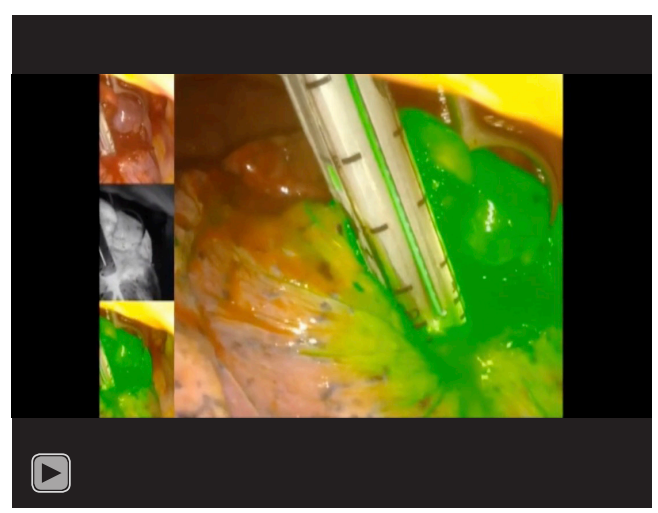

Video 3 Deep wedge resection for a patient with lung cancer at left S10c. Ten $\mathrm{mL}$ of 10 -folded diluted ICG was injected into left S10ciayx and S10aii , respectively and deep wedge resection underwent along to the border of fluorescence.

in 5 , simple segmentectomy which was defined a simple plane cut surface of pulmonary division, i.e., S6, basal, left lingual and left upper division segmentectomy, in 15 , complex segmentectomy which was defined multiple plane cut surface of pulmonary division in 16 , extended segmentectomy which was defined segmentectomy with adjacent subsegmental resection in 22 and deep wedge resection which was defined partial lung resection along with sub-segmental septum in 8 (Figure 4, Table 1).

Patient characteristics, operative data, and long-term outcomes are listed in Table 2. The average number of virtual segmentectomies performed in each case was $4.5 \pm 1.6$. The shortest distances from the tumor to the resection margin by simulation and actual measurement were $20.8 \pm 11.1$ and $22.6 \pm 8.3 \mathrm{~mm}$, respectively $(\mathrm{P}=0.186)$. This suggests that simulation was well matched with the actual operation. Average operation time was $204.3 \pm 57.7 \mathrm{~min}$, and average blood loss was $131 \pm 213 \mathrm{~mL}$.

Postoperative recurrence was found in 9 cases (distant in 8 and mediastinal lymph node in 1). No local recurrence was found in all cases.

\section{Discussion}

The use of ICG and an infrared thoracoscope in order to identify the borderline of lung resection has been reported by several authors $(7,8)$. Since their methods are mainly intravenous injection of ICG, visualization of fluorescence can continue only for a couple of minutes. The advantages of our method are (I) applicable to any type of sublobar resection, (II) initial determination of resection area at 


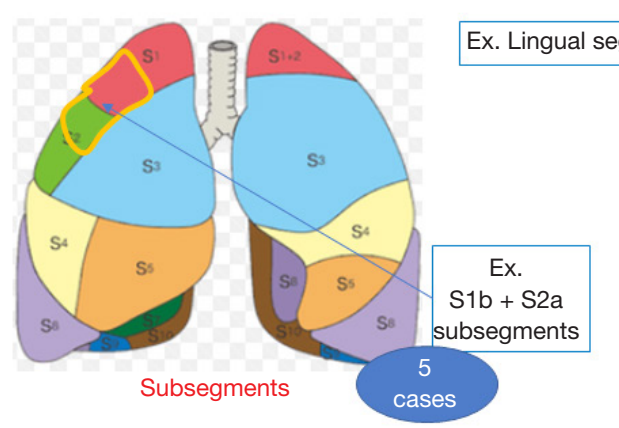

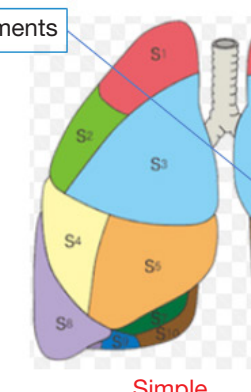

One cut surface of

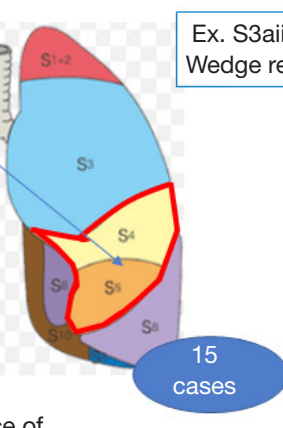

cases i.e., S6, lingual, basal, upper divisio

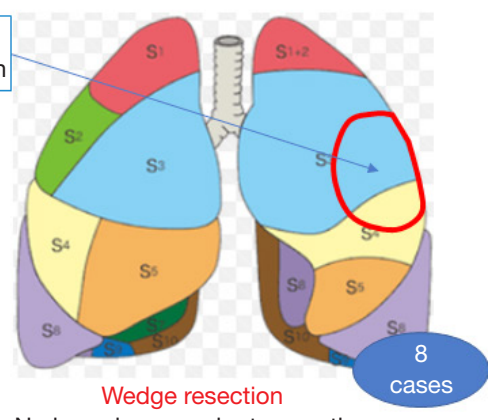

No broncho-vascular transection
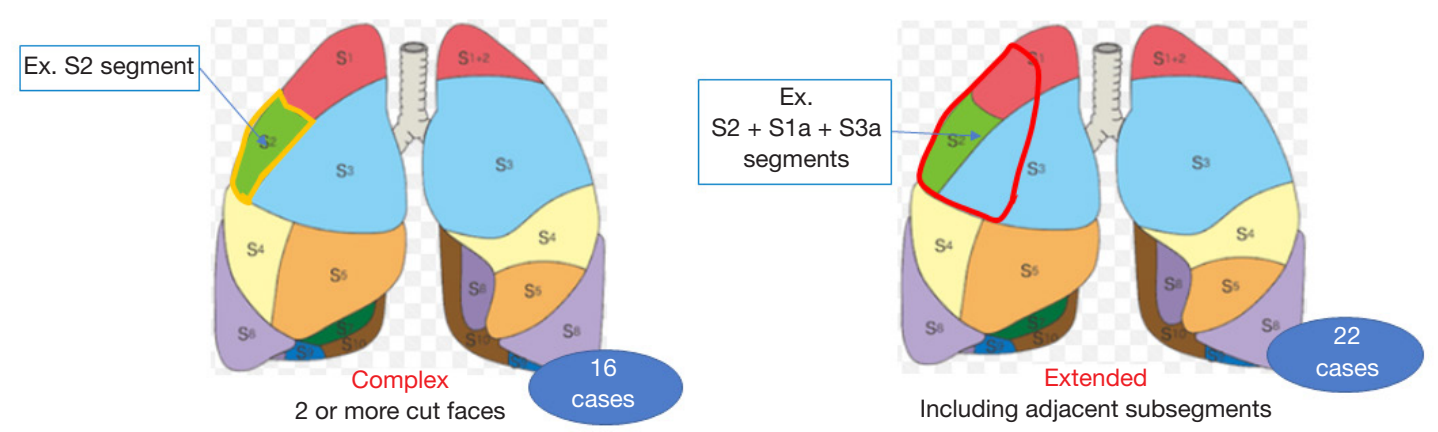

Figure 4 Sixty-six cases of ICG sublobar resection were performed, including 5 cases of subsegmental resection, 15 cases of simple segmentectomy, 16 cases of complex segmentectomy, 22 cases of extended segmentectomy, and 8 cases of anatomical super deep wedge resection. ICG, indocyanine green.

Table 1 The types of sublobar resections

Wedge resection (8 cases)

rt. S1ai+S2aii+S2bi, rt. S10ai+ii, rt. S1bi+ii, rt. S9bi+ii, It. S(1+2)ci+ii, It. S1+2cii+S3ai+S4ai, It. S4a+S1+2c+S3a, It. S8a

Subsegmentectomy (5 cases)

rt. S2b, rt. S3b, It. $S(1+2) a+b$, It. $S(1+2) b$, It. $S 10 b+c$

Simple segmentectomy (15 cases)

rt. $S 6 \times 6$ cases, rt. basal, It. upper division, It. lingual $\times 4$ cases, It. S6 22 cases, It. basal

Complex segmentectomy (16 cases)

rt. $\mathrm{S} 1 \times 2$ cases, rt, $\mathrm{S} 1+\mathrm{S} 2 \times 2$ cases, rt . S2, rt. S3 22 cases, rt. S8+S9, rt. S10 2 cases, rt. S7+S8+S9, It. S(1+2) x 3 cases, It. S8+S9 2 cases

\section{Extended segmentectomy (22 cases)}

rt. $\mathrm{S} 1+\mathrm{S} 2 \mathrm{a}+\mathrm{S} 3 \mathrm{bi}$, rt. $\mathrm{S} 1 \mathrm{a}+\mathrm{S} 2 \times 4$ cases, rt. $\mathrm{S} 2+\mathrm{S} 1 \mathrm{a}+\mathrm{S} 3 \mathrm{a}$, rt. $\mathrm{S} 2+\mathrm{S} 3 \mathrm{a}, \mathrm{rt.} \mathrm{S} 6+\mathrm{S} 10 \mathrm{a} \times 2$ cases, rt. $\mathrm{S} 6+\mathrm{S} 8 \mathrm{a}+\mathrm{S} 9 \mathrm{a}, \mathrm{rt} . \mathrm{S} 8+\mathrm{S} 9+\mathrm{S} 10 \mathrm{~b}+\mathrm{c}$, $\mathrm{S} 8+S 9 b \times 2$ cases, rt. Basal+S10*, It. $S(1+2)+S 3 a \times 2$ cases, It. S10+S9a, It. S10+S9a+S10*+S6c, It. S6+S10a, It S6+S10a+S10* 2 cases, It. S9a+S10 S10*: accessory segment of S10, which exists between S6b and S10a

operation, (III) possible deep wedge resection with enough margin without individual broncho-vascular transection, (IV) only transection of vessels and bronchi heading for removal lung and $(\mathrm{V})$ long identification of fluorescence. On the other hand, the drawbacks are (I) the necessity of a near infrared thoracoscopy and 3D medical image 
Table 2 Summary of operative procedures

\begin{tabular}{|c|c|}
\hline Characteristics & Data \\
\hline Number & 66 \\
\hline Age & $69.0 \pm 10.7$ \\
\hline Gender (M/F) & $39 / 27$ \\
\hline Tumor diameter (mm) & $21.0 \pm 11.1$ \\
\hline Op. time (min) & $204.3 \pm 57.7$ \\
\hline Blood loss (g) & $131 \pm 213$ \\
\hline \multicolumn{2}{|l|}{ Disease } \\
\hline Lung cancer & 42 \\
\hline Metastatic tumor & 21 \\
\hline Benign disease & 3 \\
\hline Average number of virtual segmentectomy & $4.5 \pm 1.6$ \\
\hline \multicolumn{2}{|l|}{ Type of sublobar resection } \\
\hline Deep wedge resection & 8 \\
\hline Subsegment & 5 \\
\hline Simple & 15 \\
\hline Complex & 16 \\
\hline Extended & 22 \\
\hline \multicolumn{2}{|l|}{ Surgical margin } \\
\hline Virtual (mm) & $21.0 \pm 11.1$ \\
\hline Actual (mm) & $22.6 \pm 8.3$ \\
\hline Postop. Complications (no/yes) & May-61 \\
\hline Prolonged air leak & 4 \\
\hline Pneumonia & 1 \\
\hline Recurrence site (no/yes) & Sep-57 \\
\hline Cutting line & 0 \\
\hline Same lobe & 0 \\
\hline Ipsilateral & 1 \\
\hline Hilar or mediastinal LN & 1 \\
\hline N3 & 1 \\
\hline Dissemination & 1 \\
\hline Distant & 4 \\
\hline Death & 5 \\
\hline Cancer death & 2 \\
\hline Other causes & 2 \\
\hline
\end{tabular}

analyzer, (II) knowledge of precise bronchial anatomy, (III) advanced manipulation skills of bronchoscopy, (IV) initial ununiformed distribution of ICG and distribution of ICG into the adjacent area with the passage of time.

We compared negative staining by i.v. ICG injection and positive staining by transbronchial ICG instillation (Table 3). Negative staining is easy to perform, with clear visibility, a stable technique, and low invasiveness. Although the visibility window of the border is very short, ICG injection can be repeated. However, all body tissues are stained and the border can be difficult to identify. On the other hand, positive staining can maintain ICG fluorescence for several hours, allowing for persistent visualization of the boundary line. The most critical and important part of positive staining is transbronchial instillation of ICG. When the transbronchial instillation is successful, the ICG border is continuously visible, making complicated sublobar resection much easier. Furthermore, when we hesitate to decide whether small vessels and bronchi should be transected, we can only treat them with heading for removal tissues. This is a significant advantage in complicated sublobar resection. However, if ICG is instilled into the wrong bronchus, surgery may be difficult and confusing because re-challenge is impossible.

Volume Analyzer Synapse 3D VINCENT is a highly advanced technology for visualizing 3D organ structures (9). It allows us to perform a simulation before the operation and minimize excision volume. However, it can be difficult to precisely match the operation with the simulation. Transbronchial ICG-sublobar resection is an ideal procedure to bridge this gap.

Intentional segmentectomy has been recognized as a surgical option for early stage lung cancer. Tsutani and colleagues reported that overall survival and recurrence free survival after segmentectomy were equivalent to lobectomy in stage IA lung adenocarcinoma (10). Cao and colleagues also reported a meta-analysis of intentionally selected segmentectomy showing similar long-term results as lobectomy. In this analysis, tumors were smaller than $2 \mathrm{~cm}$, located peripherally, and had favorable histopathology, such as lepidic adenocarcinoma and minimally invasive adenocarcinoma, with ground-glass opacities on imaging (11). Sakairi and colleagues investigated intrapulmonary spread patterns, such as segmental/intersegmental node metastasis and pulmonary parenchymal metastasis, in patients who underwent resection of two or more pulmonary segments for primary lung cancer, and concluded that metastasis outside tumor-bearing segments was rarely observed 
Table 3 Comparison between intravenous and transbronchial ICG injection

\begin{tabular}{|c|c|c|}
\hline Features & Intravenous & Transbronchial (negative staining) \\
\hline Clear visibility & (-) & $(0 \sim 0$ \\
\hline Stable technique & () & (O) $\sim \triangle$ \\
\hline Low invasiveness & () & 0 \\
\hline Uniformity of ICG & () & $(0) \sim \triangle$ \\
\hline
\end{tabular}

ICG, indocyanine green. (), highly effective; $\bigcirc$, effective; $\triangle$, not well effective.

in cases with tumors (I) without extrapulmonary nodal metastasis and (II) with ground glass opacity or peripheral small (20 mm or less) features (12). This suggests that intentional sublobar resection for peripheral nodules $2 \mathrm{~cm}$ or less in diameter may be an ideal indication for active limited resection. Our operative indication for active limited resection was the same as these investigators. However, the previous group did not describe what types of sublobar resection should be done. Sawabata reviewed locoregional recurrence after intentional pulmonary segmentectomy for NSCLC (13). He suggested that a sufficient margin distance is crucial to reducing locoregional recurrence. Schuchert and colleagues reported that anatomic segmentectomies for stage I NSCLC resulted in a higher rate of recurrence when margin/tumor ratios were less than one (14). This suggests that a $2 \mathrm{~cm}$ or greater surgical margin is necessary for early stage lung cancer. In our study, we observed no locoregional recurrence in patients with either active or passive limited resection. There were also no locoregional recurrences in the control group. This was because, compared to ICG group, a wider segmental resection was obtained (i.e., a relatively simple segmentectomy) to secure a sufficient surgical margin.

In passive limited resection, sublobar resection had a limited surgical effect because advanced lung cancer was identified in compromised patients. At the very least, locoregional recurrence at the cutting line or within the same lobe should be prevented in such cases. Our procedure could prevent locoregional recurrence, with the exception of lymph node metastasis.

The deep wedge resection by using our method is a new approach to small lung nodules. The structure of the lung is like an orange. A cluster of orange is separated from the adjacent cluster by a thin membrane. The pulmonary segmental planes are like a membrane. Each segment and subsegment is separated by an intersegmental septum, and pulmonary vessels and bronchi do not cross the septum. Therefore, when the intersegmental plane is given bilateral traction and cut by electric cautery, the lung is naturally divided along the plane. Little bleeding or air leakage is observed. This makes an anatomical super deep wedge resection along the septum possible. The associated small vessels and bronchi can be stapled at the deepest portion.

After creating a virtual sublobar resection by selecting a sub-segmental bronchus, which follows the fourth bronchial bifurcation, ICG is instilled into the associated bronchi. ICG distributes to all downstream tissues. The nodule should be in the center of this tissue. Therefore, this method serves to both localize the tumor and identify the anatomical resection region that ensures a sufficient surgical margin. The indications for deep wedge resection include tumors located in the intermediate lung with difficult resection due to compromised patient status including low cardiopulmonary function, nonpalpable tumors in the peripheral or intermediate lung, and avoidance of complete pneumonectomy for secondary tumors after upper lobectomy.

In conclusion, the combination of virtual sublobar resection and ICG-guided sublobar resection using transbronchial ICG injection is applicable to any type of sublobar resection and can prevent local recurrence of lung neoplasms. This is a novel approach to early stage lung cancers and metastatic lung tumors.

\section{Acknowledgments}

Funding: None.

\section{Footnote}

Provenance and Peer Review: This article was commissioned 
by the Guest Editor (Meinoshin Okumura) for the series "Dedicated to the 36th Annual Conference of Japanese Association for Chest Surgery (JACS)" published in Fournal of Visualized Surgery. This article has undergone external peer review.

Conflicts of Interest: All authors have completed the ICMJE uniform disclosure form (available at https://jovs.amegroups. com/article/view/10.21037/jovs.2019.12.12/coif). The series "Dedicated to the 36th Annual Conference of Japanese Association for Chest Surgery (JACS)" was commissioned by the editorial office without any funding or sponsorship. The authors have no other conflicts of interest to declare.

Ethical Statement: The authors are accountable for all aspects of the work in ensuring that questions related to the accuracy or integrity of any part of the work are appropriately investigated and resolved. The study was conducted in accordance with the Declaration of Helsinki (as revised in 2013). The study protocol was approved by the Research Ethics Board of Tokyo Women's Medical University, Japan. Written informed consent was obtained from all patients.

Open Access Statement: This is an Open Access article distributed in accordance with the Creative Commons Attribution-NonCommercial-NoDerivs 4.0 International License (CC BY-NC-ND 4.0), which permits the noncommercial replication and distribution of the article with the strict proviso that no changes or edits are made and the original work is properly cited (including links to both the formal publication through the relevant DOI and the license). See: https://creativecommons.org/licenses/by-nc-nd/4.0/.

\section{References}

1. Landreneau RJ, Normolle DP, Christie NA, et al. Recurrence and survival outcomes after anatomic segmentectomy versus lobectomy for clinical stage I nonsmall-cell lung cancer: a propensity-matched analysis. J Clin Oncol 2014;32:2449-55.

2. Koike T, Yoshiya K, Tsuchida M, et al. Risk factor analysis of locoregional recurrence after sublobar resection in patients with clinical stage IA non-small cell lung cancer. J Thorac Cardiovasc Surg 2013;146:372-8.

3. Sekine Y, Ko E, Oishi H, et al. Simple and effective technique for identification of intersegmental planes by infrared thoracoscopy after transbronchial injection of indocyanine green. J Thorac Cardiovasc Surg 2012;143:1330-5.

4. Jafek BW, Carter DR. Endoscopic nomenclature for bronchopulmonary anatomy. Otolaryngol Head Neck Surg 1979;87:815-7.

5. Nomina Anatomica. 6th edition. New York: Churchill Livingstone, 1989.

6. Kanazaki K, Sano K, Makino A, et al. Development of human serum albumin conjugated with near-infrared dye for photoacoustic tumor imaging. J Biomed Opt 2014;19:96002.

7. Misaki N, Chang SS, Gotoh M, et al. A novel method for determining adjacent lung segments with infrared thoracoscopy. J Thorac Cardiovasc Surg 2009;138:613-8.

8. Mun M, Nakao M, Matsuura Y, et al. Thoracoscopic segmentectomy for small-sized peripheral lung cancer. J Thorac Dis 2018;10:3738-44.

9. Oshiro Y, Ohkohchi N. Three-Dimensional Liver Surgery Simulation: Computer-Assisted Surgical Planning with Three-Dimensional Simulation Software and ThreeDimensional Printing. Tissue Eng Part A 2017;23:474-80.

10. Tsutani Y, Miyata Y, Nakayama H, et al. Oncologic outcomes of segmentectomy compared with lobectomy for clinical stage IA lung adenocarcinoma: propensity score-matched analysis in a multicenter study. J Thorac Cardiovasc Surg 2013;146:358-64.

11. Cao C, Chandrakumar D, Gupta S, et al. Could less be more?-A systematic review and meta-analysis of sublobar resections versus lobectomy for non-small cell lung cancer according to patient selection. Lung Cancer 2015;89:121-32.

12. Sakairi Y, Yoshino I, Yoshida S, et al. Pattern of metastasis outside tumor-bearing segments in primary lung cancer: rationale for segmentectomy. Ann Thorac Surg 2014;97:1694-700.

13. Sawabata N. Locoregional recurrence after pulmonary sublobar resection of non-small cell lung cancer: can it be reduced by considering cancer cells at the surgical margin? Gen Thorac Cardiovasc Surg 2013;61:9-16.

14. Schuchert MJ, Pettiford BL, Keeley S, et al. Anatomic segmentectomy in the treatment of stage I non-small cell lung cancer. Ann Thorac Surg 2007;84:926-32; discussion 932-3.

doi: 10.21037/jovs.2019.12.12

Cite this article as: Sekine Y, Koh E, Oheda H, Hoshino H. The new technology of precise anatomical sublobar resection by using 3D medical image analyzer and a fluorescence guided surgery with transbronchial injection of indocyanine green. J Vis Surg 2020;6:41. 